\title{
Tonsillar Fossa
}

National Cancer Institute

\section{Source}

National Cancer Institute. Tonsillar Fossa. NCI Thesaurus. Code C12235.

A triangular shaped space that lies on each side of the oropharynx and houses the palatine tonsils. 